\title{
NON-COMMUTATIVE INDEPENDENCE OF ALGEBRAS AND APPLICATIONS TO PROBABILITY
}

\author{
JANUSZ WYSOCZAŃSKI
}

Abstract. We present the notions of independence, which appear in non-commutative probability. The basic ones are free, boolean and monotonic independences, formulated for families of algebras indexed by totally ordered set. A generalization of the latter two is the bm-independence, defined for partially ordered index sets. For each independence there is an analogue of the classical central limit theorem. In the case of bm-independence this depends also on the index set. Examples of such partially ordered index sets are discrete lattices in symmetric positive cones.

Mathematics subject classification (2010): 46L54, 81R50.

Keywords and phrases: Monotonic and boolean independence, partially ordered sets, non-commutative probability, limit theorems, partitions, graphs.

\section{REFERENCES}

[1] D. Avitzour, Free Product of $C^{*}$-Algebras, Trans. Amer. Math. Soc., 271, 2 (1982), $423-435$.

[2] M. BoŻEJKO, Positive definite functions on the free group and the non-commutative Riesz product, Boll. Un. Mat. Ital., 6, 5A (1986), 13-21.

[3] J. Faraut, A. Koranyi, Analysis on Symmetric Cones, Oxford Univ. Press 1994.

[4] U. Franz, Monotone independence is associative, Infin. Dimens. Anal. Quantum Probab. Relat. Top., 4, 3 (2001), 401-407.

[5] Y. G. LU, An interacting Fock space and the arcsine law, Probab. Math. Stat., 17 (1997), 149-166.

[6] N. Muraki, A new example of non-commutative "de Moivre-Laplace theorem", Prob.Th. Math. Stat., Proceedings 7th Japan-Russia Symposium, Tokyo 1995, ed. Watanabe, S. et al., World Scientific (1996), 353-362.

[7] N. MURAKI, Monotonic independence, monotonic central limit theorem and monotonic law of small numbers, Infin. Dimens. Anal. Quantum Probab. Relat. Top., 4, 1 (2001), 39-58.

[8] D. VOICULESCU, Symmetries of some reduced free product $C^{*}$-algebras, in Operator algebras and their connections with topology and ergodic theory (Buşteni, 1983), 556-588, Lecture Notes in Math., 1132, Springer, Berlin, 1985.

[9] J. Wysoczański, Monotonic independence on weakly monotone Fock space, Inf. Dim. Anal. Quantum Prob. Rel. Top., 8, 2 (2005), 259-275.

[10] J. WysocZAŃsKI, Monotonic independence associated with partially ordered sets, Infin. Dimens. Anal. Quantum Probab. Relat. Top., 10, 1 (2007), 17-41.

[11] J. Wysoczańs KI, bm-Central Limit Theorems for Positive Definite Real Symmetric Matrices, Infin. Dimens. Anal. Quantum Probab. Relat. Top., 11, 1 (2008), 1-19. 